\title{
A Restoration Method for Images Including Lost Pixels based on A Similarity between A Local Region and A Neighbor Region of A Lost Pixel
}

\author{
Hiroki Imamura, Makoto Fujimura and Hideo Kuroda
}

\begin{abstract}
In the field of image restoration of unnecessary regions, a method based on optical flow has been proposed. This method can accurately restore unnecessary regions in case of changing of edge direction or existence of plural edges in unnecessary regions. However, when continuity of intensity between a pixel in unnecessary regions and its neighboring pixels is not satisfied, the method can not accurately restore unnecessary regions. In this paper, to solve the problem, we propose a method based on similarity between a local region and a neighboring region of a lost in unnecessary regions.
\end{abstract}

\section{INTRODUCTION}

There are some cases that we would like to remove unnecessary regions such as scratches in old films or subtitles. In that case, it is necessary to restore such regions as if there were not in there. To do such process by a computer, some methods have been proposed [1]-[5].

In restoration of unnecessary regions, precise restoration in edge regions is an important problem. To solve the problem, some methods have been proposed[7]-[9]. By interpolation of intensities of images, using NURBS curves, and using edge detection based on Hough transformation, Park et al. [7] proposed a restoration method which can precisely restore intensity of unnecessary regions in edge parts. Shirani et al. [8] proposed a restoration method which can precisely restore intensity of unnecessary regions in edge parts using MRF model [11]. These methods can precisely restore intensity only when there is the only one edge in a unnecessary region. However, when there are at least two edges or direction of edges abruptly changes in a unnecessary region, it is difficult to restore intensities precisely by those method. To solve the problem, Ogawa et al. [9] proposed a restoration method based on optical flow used for image sequence analysis. By this method, the above problems have been solved. However, In the regions where intensities of image abruptly change, we can not restore intensities of unnecessary regions by the method, because the method based on the correlation of intensity between intensity in a restored pixel and its neighboring pixels.

H. Imamura is with Faculty of Computer and Information Science, Department of Engineering, Nagasaki University, 1-14 Bunkyou-mach Nagasaki City, Japan imamura@cis.nagasaki-u.ac.jp

M. Fujimura is with Faculty of Computer and Information Science, Department of Engineering, Nagasaki University, 1-14 Bunkyou-mach Nagasaki City, Japan makoto@cis.nagasaki-u.ac.jp

H. Kuroda is with Faculty of Graduate School of Science and Technology, Nagasaki University, 1-14 Bunkyou-mach Nagasaki City, Japan kuroda@cis.nagasaki-u.ac.jp
In this paper, we propose a restoration method which has the same property of Ogawa's method and can precisely restore unnecessary regions such as texture regions where intensities of image abruptly change. To realize the method, we take an approach that we focus on the relationship of intensity on a pixel and its neighboring pixels, in other words, we focus on the property of fractal of images [10].

There is a restoration method based on the property of fractal of images [6]. Because method restores unnecessary regions by using only large square regions which are similar to the unnecessary region, sense of incongruity is caused in boundaries of unnecessary regions and its neighboring regions.

On the other hand, we propose a restoration method based on not only the property of fractal of images but also Ogawa's method. By this approach, we can expect that the proposed method can precisely restore unnecessary regions where intensities of images change abruptly and natural restoration in boundaries of unnecessary regions and its neighboring regions. By this approach, we can expect that the proposed method can precisely restore unnecessary regions where intensities of images change abruptly, and can actualize natural restoration in boundaries of unnecessary regions and its neighboring regions.

\section{THE ALGORITHM OF THE PROPOSED METHOD}

The method proposed by Ogawa et al. [9] restores unnecessary regions in a image by choosing a pixel similar to the neighboring pixel of a pixel in the unnecessary regions. This approach is remarkably effective when the intensities between a pixel in the unnecessary regions and its neighboring pixels smoothly change spatially. However, that method can not precisely restore unnecessary regions when the intensities between a pixel in the unnecessary regions and its neighboring pixels remarkably change spatially, such as texture regions [9] .

First, we show the approach of the proposed method for solving the above problem. Next, we show the algorithm of the proposed method.

\section{A. The approach of the proposed method}

To solve the above problem, we focus on fractal property in images [10]. The fractal property means there are some regions similar to a local region in a image. According to the fractal property, there are some regions similar to a unnecessary region in a image. Therefore, we can expect 


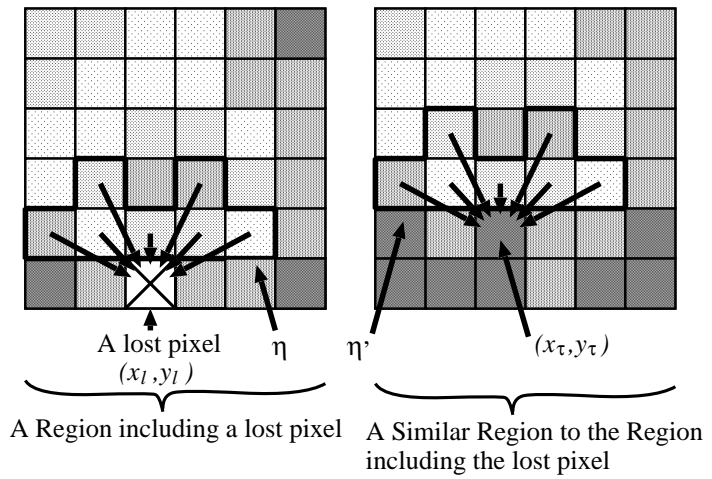

Fig. 1. The approach of the proposed method.

that there are some region similar to a region including an unnecessary region. Moreover, in that region, there some local regions $\eta^{\prime}$ similar to a neighboring region $\eta$ of a pixel in an unnecessary reigon. Furthermore, in $\eta^{\prime}$, we can expect that the intensity $I\left(x_{\tau}, y_{\tau}\right)$ in the pixel $\left(x_{\tau}, y_{\tau}\right)$ corresponding to $\left(x_{l}, y_{l}\right)$ in $\eta$ corresponds to the intensity $I\left(x_{l}, y_{l}\right)$ in the pixel $I\left(x_{l}, y_{l}\right)$. If this relation holds, whether the intensities of a pixel in unnecessary regions and its neighboring pixel smoothly change or not, we can precisely restore unnecessary regions (fig.1). Therefore, we can expect that, even if intensities in unnecessary regions and its neighboring regions remarkably change, unnecessary regions can be restored by the proposed method. We restore unnecessary regions by the following 2 steps based on fractal property of images.

As STEP1, we extract a global region similar to the region including $\left(x_{l}, y_{l}\right)$. As STEP2, in the extracted region, we extract a local region $\eta^{\prime}$ similar to the neighboring region $\eta$ of a pixle in unnecessary regions. We then assign $I\left(x_{\tau}, y_{\tau}\right)$ of $\left(x_{\tau}, y_{\tau}\right)$ in $\eta^{\prime}$, which is corresponding to $\left(x_{l}, y_{l}\right)$ in $\eta$, to $\left(x_{l}, y_{l}\right)$ in $\eta$. In STEP1, we globally extract a similar region of a unnecessary region in a whole image. In STEP2, we locally extract a similar region of a unnecessary region in the region extracted in STEP1. By these 2 steps, we can expect that the proposed method can precisely restore intensity on pixels of unnecessary regions in the boundary between an unnecessary region and its neighboring region, without sense of incongruity.

Figure 2 shows the flow of the whole processing in the proposed method. In this paper, the unnecessary regions are decided in advance by manual, as well as other restoration methods.

\section{B. The algorithm of the proposed method}

1) STEP 1:Extraction of a resemblance image region of a region including an unnecessary region: First, we extract a pixel $\left(x_{l}, y_{l}\right)$ of an unnecessary region by raster-scanning on the image whose size is $X \times Y$ [pixel]. Next, we set an image region $R_{l}\left(x_{l}, y_{l}\right)$, whose size is $L \times L$ [pixel] (fig.3), including a pixel $\left(x_{l}, y_{l}\right)$ of an unnecessary region. Finally, we extract a region (a resemblance image region) resembles

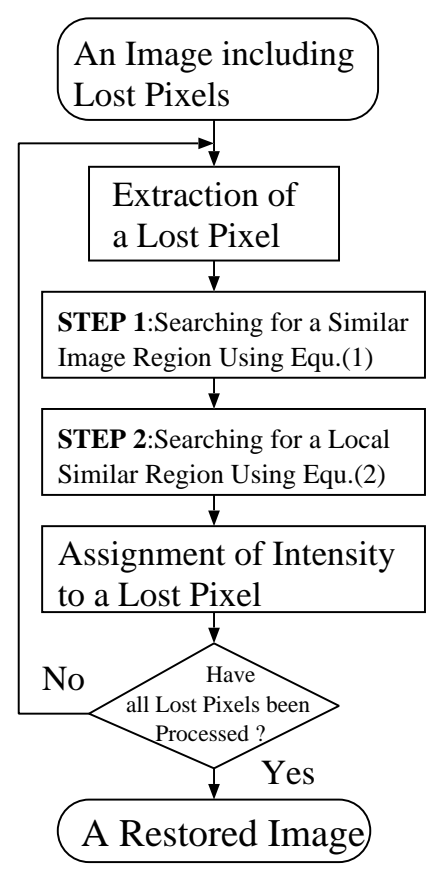

Fig. 2. Flow of the processing in the proposed method.

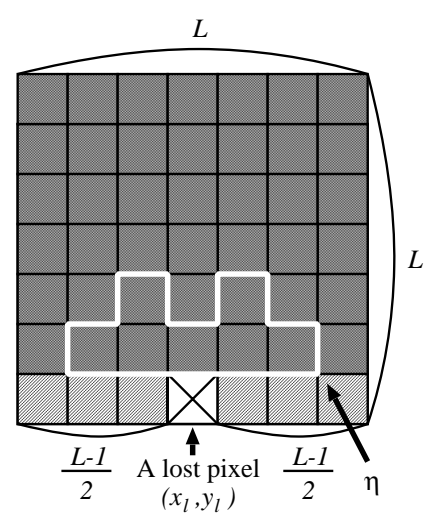

Fig. 3. The setting \#1 of a image region $R_{l}\left(x_{l}, y_{l}\right)$ and the local region.

$R_{l}\left(x_{l}, y_{l}\right)$, which is not including an unnecessary region. To extract a resemblance image region, we set the pixel $\left(x^{\prime}, y^{\prime}\right)$ corresponding to $\left(x_{l}, y_{l}\right)$ shown in fig.3. We extract an image region which satisfies

$$
R_{s}\left(x_{l}, y_{l} ; x^{\prime}, y^{\prime}\right)=\arg \min _{\substack{x^{\prime} \in R_{x^{\prime}} \\ y^{\prime} \in R_{y^{\prime}}}} \frac{1}{N_{L}} \sum_{p, q \in D_{L}} A B S_{I},
$$

where, $R_{x^{\prime}}, R_{y^{\prime}}$ denote ranges of $x^{\prime}, y^{\prime}$, where resemblance image regions can be obtained, respectively, $D_{L}$ denotes the region described as dense textured region in fig. $3, N_{L}$ denotes the number of pixels in the region described as dense textured region in fig. $3, A B S_{I}=\mid I\left(x_{l}+p, y_{l}+q\right)-I\left(x^{\prime}+\right.$ $\left.p, y^{\prime}+q\right) \mid, I\left(x_{l}+p, y_{l}+q\right), I\left(x^{\prime}+p, y^{\prime}+q\right)$ denote the intensity in $\left(x_{l}+p, y_{l}+q\right),\left(x^{\prime}+p, y^{\prime}+q\right)$, respectively, 


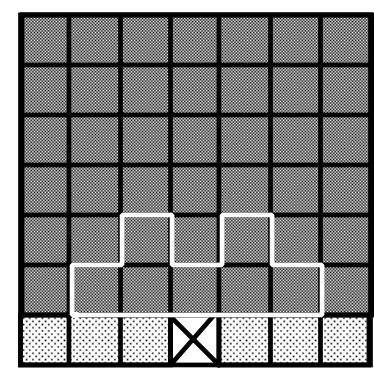

$k=1$

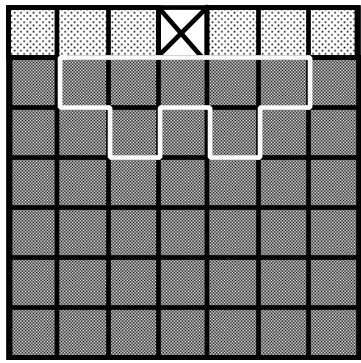

$k=5$

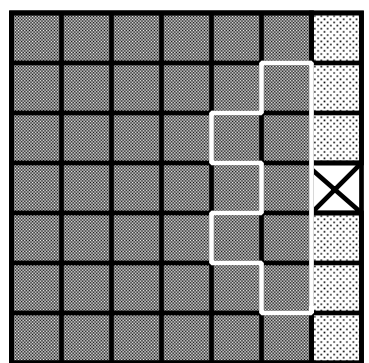

$k=3$

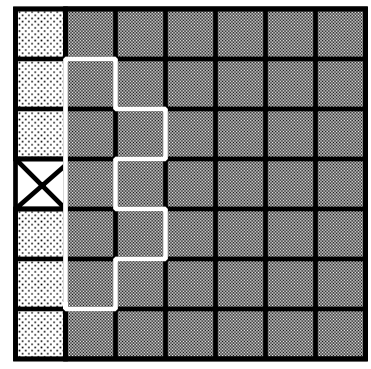

$k=7$
Fig. 4. The setting of a image region $R_{l}\left(x_{l}, y_{l}\right)$ and the local region $\eta(k=1,3,5,7)$.

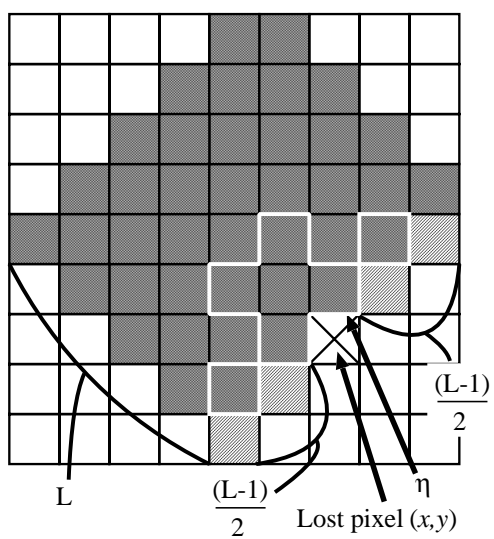

Fig. 5. The setting \#2 of a image region $R_{l}\left(x_{l}, y_{l}\right)$ and the local region $\eta$.

$R_{s}\left(x_{l}, y_{l} ; x^{\prime}, y^{\prime}\right)$ denotes the resemblance image region of $R_{l}\left(x_{l}, y_{l}\right)$.

2) STEP2:Extraction of a local resemblance image region in the resemblance image region: Next, in $R_{s}\left(x_{l}, y_{l} ; x, y^{\prime}\right)$, we extract a local resemblance image region $\eta^{\prime}$ resembles $\eta$ (fig.3) in $R_{l}\left(x_{l}, y_{l}\right)$. We extract $\eta^{\prime}$ by using

$$
\arg \min \frac{1}{7} \sum_{\substack{r, s \in \eta \\ t, u \in \eta^{\prime} \\ v \in R_{s}\left(x_{l}, y_{l} ; x, y^{\prime}\right)}} A B S_{e},
$$

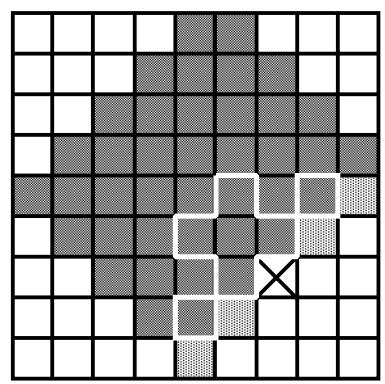

$k=2$

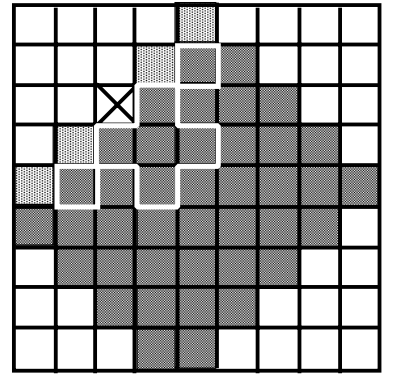

$k=6$

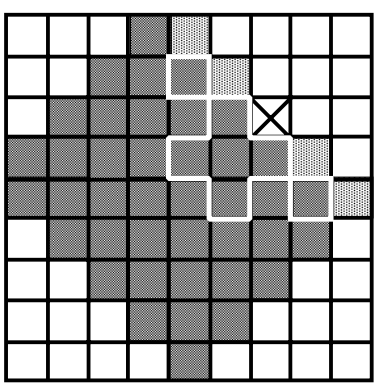

$k=4$

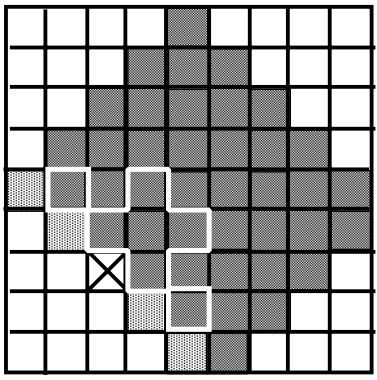

$k=8$
Fig. 6. The setting of a image region $R_{l}\left(x_{l}, y_{l}\right)$ and the local region $\eta$.

where, $A B S_{e}=\left|I\left(x_{l}+r, y_{l}+s\right)-I(v+t, w+u)\right|$, $I\left(x_{l}+r, y_{l}+s\right), I(v+t, w+u)$ denote intensities in $\left(x_{l}+r, y_{l}+s\right),(v+t, w+u)$ respectively. We set the direction of $\eta^{\prime}$ to 8 directions, The settings of $\eta^{\prime}$ is to rotate $\eta$ shown in fig. 3 every $\frac{(k-1)}{4} \pi(k=1,2, \cdots, 8)$. When the angle of rotations are $\frac{(k-1)}{4} \pi(k=1,3,5,7)$, we rotate $\eta$ shown in fig. 3 according to fig. 4 respectively. When the angle of rotations are $\frac{(k-1)}{4} \pi(k=2,4,6,8)$, we rotate $\eta$ shown in fig.5 according to fig.6 respectively. Finally, by assigning $I\left(x_{\tau}, y_{\tau}\right)$ on $\left(x_{\tau}, y_{\tau}\right)$ in $\eta^{\prime}$ satisfies equ.(2), to $\left(x_{l}, y_{l}\right)$ in $\eta$, we restore $I\left(x_{l}, y_{l}\right)$ on $\left(x_{l}, y_{l}\right)$.

3) Acquisition of the final restored image: In the proposed method, the error in estimated intensity affects the intensity to be estimated in later processing. Therefore, in 8 directions, we estimate restored images $\operatorname{Img}_{k}(x, y)(k=$ $1,2, \cdots, 8)$ by changeing direction of raster-scanning. The direction of raster-scanning is to be rotated every $\frac{(k-1)}{4} \pi(k=$ $1,2, \cdots, 8)$. The window for extraction of a resemblance image region is to be rotated every $\frac{(k-1)}{4} \pi(k=1,2, \cdots, 8)$, as the center of rotation is $(x, y)$ of $R_{l}(x, y)$ and $\left(x^{\prime}, y^{\prime}\right)$ of $R_{s}\left(x, y ; x^{\prime}, y^{\prime}\right)$ respectively. When the angle of rotations are $\frac{(k-1)}{4} \pi(k=1,3,5,7)$, According to fig. 4 , we rotate the the regions described as dense textured regions in fig. 3 When the angle of rotations are $\frac{(k-1)}{4} \pi(k=2,4,6,8)$, According to fig.6, we rotate the regions described as dense textured regions in fig.5. In this case, $D_{L}$ と $N_{L}$ in equ.(1) denote the number of pixels in the regions described as dense textured regions in fig.5 respectively. The settings of $\eta$ is to be rotated the regions shown in fig. 3 every $\frac{(k-1)}{4} \pi(k=1,2, \cdots, 8)$, as well as the directions of raster-scanning. When the angle of 
rotations are $\frac{(k-1)}{4} \pi(k=1,3,5,7)$, According to fig.4, we rotate $\eta$ in fig. 3 When the angle of rotations are $\frac{(k-1)}{4} \pi(k=$ $2,4,6,8)$, According to fig.6. we rotate $\eta$ in fig.5. Finally, we acquire the final restored image $\operatorname{Img}_{\text {final }}(x, y)$ by

$$
\begin{aligned}
& \operatorname{Img}_{\text {final }}(x, y) \\
= & \operatorname{Med}\left\{\operatorname{Img}_{1}(x, y), \operatorname{Img}_{2}(x, y), \cdots, \operatorname{Img}_{8}(x, y)\right\} .
\end{aligned}
$$

\section{EXPERIMENTS}

To evaluate the effectiveness of the proposed method, we experimented with the method in literature [6], [9] and the proposed method. In this experiment, we used Barbara and Mandrill image shown in fig.7, which include texture image regions where intensities change remarkably, such as stripes or hairs. And we used Lenna and Peppers image shown in fig.7, which include flat region where intensities change smoothly. The size of the color images used in this experiment is $256 \times 256$ [pixel], and the level of color images is $8[\mathrm{bit} / \mathrm{pixel}]$. To generate the images including unnecessary regions, we randomly add 10 squared unnecessary regions, whose intensity level in $R, G$ and $B$ is 255 respectively, to the original images shown in fig.7. In the method in literature [6], we set the size of cell to $16 \times 16$ [pixels], and set the number of used vectors to 12 . In the method in literature [9], we set $L=7, \alpha=0.5$. In the proposed method, we set $L=7$, to be the best result. Since we use color images in this experiment, we used $A B S_{e}$

$$
\begin{aligned}
& A B S_{I} \\
& =\frac{1}{3} \quad\left\{\left|I_{R}(x+p, y+q)-I_{R}\left(x^{\prime}+p, y^{\prime}+q\right)\right|\right. \\
& +\left|I_{G}(x+p, y+q)-I_{G}\left(x^{\prime}+p, y^{\prime}+q\right)\right| \\
& \left.+\left|I_{B}(x+p, y+q)-I_{B}\left(x^{\prime}+p, y^{\prime}+q\right)\right|\right\}, \\
& \begin{array}{cl}
A B S_{e} & \\
\frac{1}{3} & \left\{\left|I_{R}\left(x_{l}+r, y_{l}+s\right)-I_{R}(v+t, w+u)\right|\right. \\
+ & \left|I_{G}\left(x_{l}+r, y_{l}+s\right)-I_{G}(v+t, w+u)\right| \\
+ & \left.\left|I_{B}\left(x_{l}+r, y_{l}+s\right)-I_{B}(v+t, w+u)\right|\right\}
\end{array}
\end{aligned}
$$

instead of equ.(1) and equ. (2) respectively. where $I_{R}(x, y)$, $I_{G}(x, y), I_{B}(x, y)$ denote inteisities of $R, G$ and $B$ in $(x, y)$ respectively. In this experiment, we call the method in literature [6] the method A, the method in literature [9] the method B and the proposed method the method C.

\section{A. Quantitative experiment}

To evaluate effectiveness of the proposed method quantitatively, we used

$$
P S N R=10 \log _{10} \frac{255^{2}}{M S E}[\mathrm{~dB}] .
$$

where, $M S E$ denotes

$$
M S E=\frac{1}{N_{l o}} \sum_{i=1}^{N_{l o}}\left|I_{e}^{(i)}(x, y)-I_{o}^{(i)}(x, y)\right|^{2}
$$

TABLE I

$P S N R$ OF EACH METHOD IN EACH IMAGE.

\begin{tabular}{c||c|c|c}
\hline \multicolumn{1}{c||}{ Images } & \multicolumn{3}{c}{$P S N R[\mathrm{~dB}]$} \\
\cline { 2 - 4 } & Method A & Method B & Method C \\
\hline \hline Barbara & 34.15 & 34.20 & 35.48 \\
\hline Lenna & 34.79 & 37.42 & 37.88 \\
\hline Mandrill & 34.26 & 34.78 & 35.61 \\
\hline Peppers & 37.39 & 39.03 & 39.15 \\
\hline
\end{tabular}

where, $I_{e}^{(i)}(x, y)$ denotes the intensity on $(x, y)$ in the restored image, which was $i$-th pixel in unnecessary regions, $I_{o}^{(i)}(x, y)$ denotes the intensity on $(x, y)$ corresponding $I_{e}^{(i)}(x, y)$ in the original images, $N_{l o}$ denotes the number of pixels in unnecessary regions. The experimental result is shown in table I. The PSNR shown in the table denotes 5 times average.

From the result, we see that the $P S N R$ in the proposed method is improved comparing to the other methods. The method A takes an approach based on fractal property, as well as the proposed method. However, the method extracts, as a global image region, the region similar to an unnecessary region, and restores unnecessary regions, using only the global image region. Therefore, In boundaries between an unnecessary region and its neighboring regions, which are in restored image by the method A, sense of incongruity is caused in boundaries of unnecessary regions and its neighboring regions. On the other hand, the proposed method, at first, extracts a image region similar to an unnecessary region globally, then, locally restored images including unnecessary regions as well as the method $\mathrm{C}$. Therefore, the proposed method can precisely restore images including unnecessary regions, comparing to the method C. Moreover, we see that the result of $P S N R$ in the method $\mathrm{B}$ is higher than that in the method C. Especially, in Barbara and Mandrill whose intensities remarkably change, the result of $P S N R$ in the method $\mathrm{C}$ is higher about $1[\mathrm{~dB}]$ than in the method $\mathrm{B}$. From the result, especially, the method $\mathrm{C}$ is effective for the image including texture region whose intensities remarkably change.

\section{B. Qualitative experiment}

Next, We show the qualitative experimental results by the method A, the method B and the method C. In this experiment, the parameters in each method are the same values in the quantitative experiment. Figure 9 shows the expanded images in restored images by each method, the original images and images including unnecessary regions in the square region in fig.8. In fig.9, we see that the result of the method B and C are fine. This is because intensities between a pixel in an unnecessary region and its neighboring pixel smoothly change. On the other hand, the result by the method A is not fine. This is because the method A extracts a global region similar to an unnecessary region, and uses only information of the global region. We consider that the result of Mandrill in fig.9 is the same reason. In the result of Peppers in fig.9, in an unnecessary region where plural edges go through or direction of edges remarkably changes, we see 


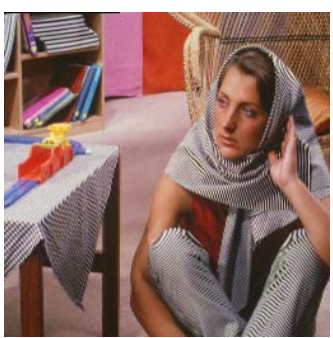

Barbara

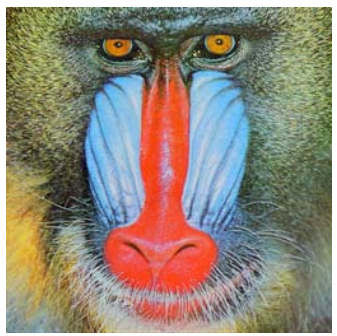

Mandrill

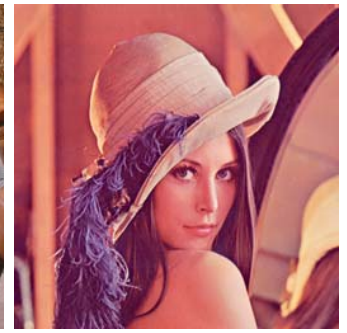

Lenna

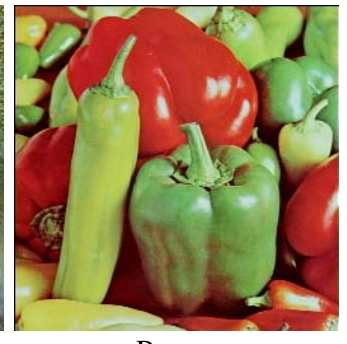

Peppers
Fig. 7. The original images of the images including lost pixels used in the experiment.

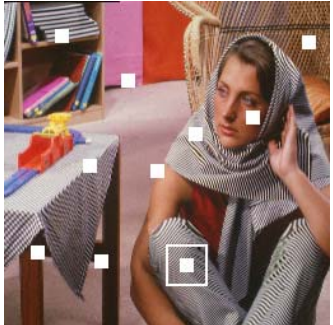

Barbara

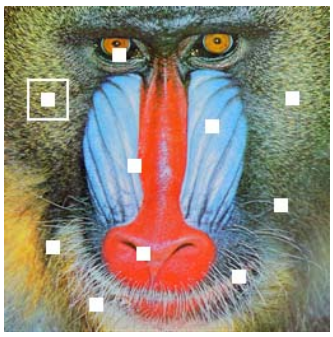

Mandrill

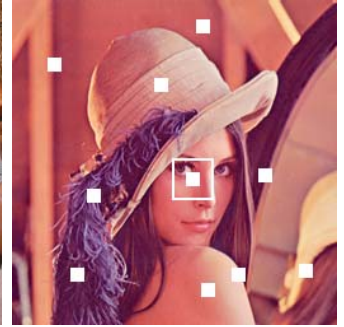

Lenna

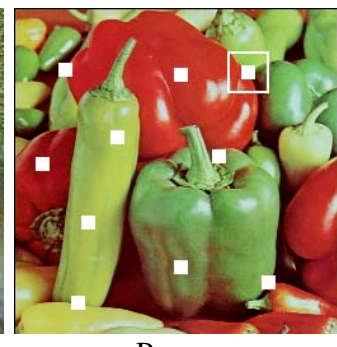

Peppers
Fig. 8. The images including lost pixels used in the experiment.

that the result of the method $\mathrm{B}$ and $\mathrm{C}$ are fine. Therefore, we consider that the proposed method has the same property as the method B. On the other hand, the result of the method A is unnatural in the intersection of edges. In the ellipse regions of Barbara and Mandrill in fig.9, unnatural lineal texture caused in the method B. This is because that the method B restores unnecessary regions by assigning an intensity of a neighboring pixel whose adaptation parameter is high. This adaptation parameter is high when its intensity is smooth spatially. Therefore, we consider that pixels in unnecessary regions are successively assigned intensities which is smooth spatially, unnatural lineal textures are generate. On the other hand, the result of the method $\mathrm{C}$ is fine. From the above results, the proposed method can precisely restore unnecessary regions where intensities remarkably change and have the same property as the method B.

\section{CONCLUSION}

To solve the problem such that the method based on optical flow can not precisely restore unnecessary regions in texture regions, we proposed the restoration method based on fractal property of global and local image regions. To evaluate the effectiveness of the proposed method, we experimented with the conventional methods and the proposed method. In the experiments, the result of the proposed method was fine, especially, in texture regions such that intensities remarkably change and in a region where plural edges go through. From the results, we see that the proposed method has the same property as the method in literature [9] and can precisely restore unnecessary regions where intensities remarkably change. In the future, by considering rotation, expansion/reduction in extraction of a local resemblance region, we expect that the accuracy of restoration by the proposed method will be improved.

\section{REFERENCES}

[1] M. Bertalmio, G. Sapiro, V.Caselles and C. Ballester," Image Inpainting, " in Proc. SIGGRAPH'00, pp.417-424, 2000.

[2] S. Masnou and J. Morel," Level Lines based Disocclusion," in Proc. IEEE int. Conf. Image Processing, vol.3, pp.259-263, 1998.

[3] A. N. Hirani and T. Totsuka, " Combining Frequency and Spatial Domain Information for Fast Interactive Image Noise Removal, " in Proc. SIGGRAPH'96, pp.269-276, 1996.

[4] L. -Y. Wei and M. Levoy, " Fast Texture Synthesis using Treestructured Vector Quantization," in Proc. SIGGRAPH'00, pp.479-488, 2000.

[5] T. Amano , Y. Sato "' Image Interpolation Uising BPLP Method on the Eigenspace," Trans. on IEICE, Vol.J85D-II, No. 3, pp.457-465, 2002.

[6] S. Izoe , Y. Kenmochi , K. Kotan ", Fractal-based Image Disocclusion using Kernel Principal Components Analysis," in Proc. IMPS'01, pp.39-40, 2001

[7] J. W. Park and U. S. Lee," Recovery of Corrupted Image data based on the NURBS Interpolation," IEEE Trans. Circuits Syst. Video Technol., Vol.9, No.7, pp.1003-1008, 1999.

[8] S. Shrani, F. Kossentini and R. Ward," A Concealment Method for Video Communications in An Errorprone Environment," IEEE J. Sel, Areas Comun., Vol.18, No.16, pp.1122-1128, 2000.

[9] T. Ogawa , M. Hasayama , H. Kitajima "' Restoration for Missing Intensity of Still Images Using the Optical Flow," Trans. on IEICE, Vol.J87D-II, No. 9, pp.1786-1795, 2004.

[10] A. E. Jacquin," Image Cording based on Fractal Theory of Interated Convractive Image Transformation," IEEE Trans. Image Process., Vol.1, No.1, pp.18-30, 1992.

[11] S. Geman and D. Geman," Stochastic Relaxation, Gibbs Distributions, and the Bayesian Relaxation of Images," IEEE Trans. Pattern Anal. Mach. Intell, Vol.PAMI-6, No.6, pp.721-741, 1984.

[12] B. K. P. Horn and B. G. Schunck," Determining Optical Flow," Artif. Intell., Vol.17, pp.185-203, 1981. 


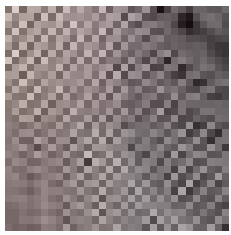

Barbara (Method A)

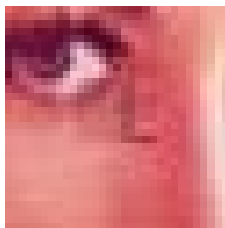

Lenna (Method A)

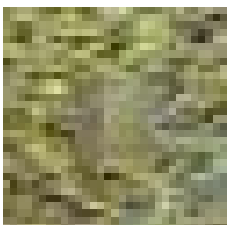

Mandrill (Method A) Mandrill (Method B)

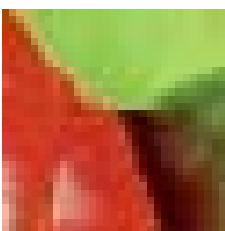

Peppers (Method A)

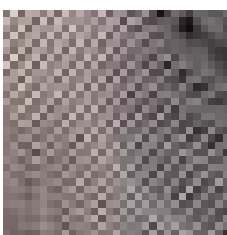

Barbara (Original)

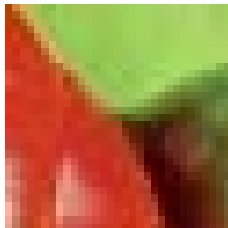

Peppers (Method B)

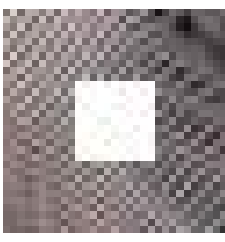

Barbara (Lost Pixels)
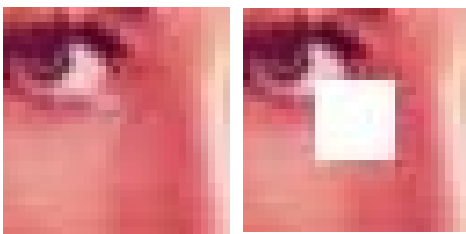

Lenna (Original)

Lenna (Lost Pixels)
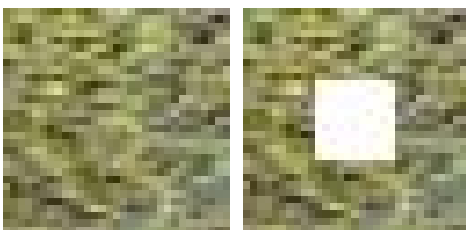

Mandrill (Original) Mandrill (Lost Pixels)
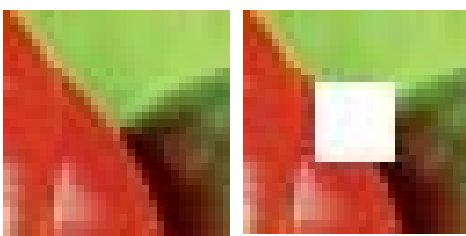

Peppers (Original)

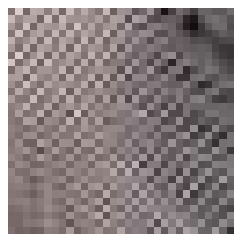

Barbara (Method C)

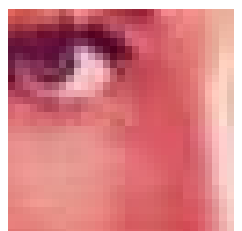

Lenna (Method C)

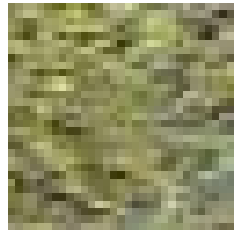

Mandrill (Method C)

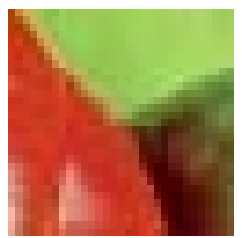

Peppers (Method C)

Fig. 9. The expanded images of the restored images by each method, the original images and the images in the rectangle region in Fig.8. 